\title{
ON THE STABILITY OF MIDPOINT SMOOTHING WITH LEGENDRE POLYNOMIALS
}

\author{
WILLIAM F. TRENCH
}

1. Introduction. Let $f$ be square integrable on $[-1,1]$, and let $p$ be the least squares polynomial fit to $f$ of degree not exceeding $2 k+1$. It is known, [1], that

$$
p(0)=\int_{-1}^{1} w(x) f(x) d x
$$

where

$$
w(x)=\frac{\left(k+\frac{1}{2}\right) P_{2 k}(0) P_{2 k+1}(x)}{x},
$$

$P_{2 k}$ and $P_{2 k+1}$ being the Legendre polynomials. Let

$$
C(\theta)=\int_{-1}^{1} w(x) \cos x \theta d x .
$$

The principal result of this paper is the following theorem.

Theorem 1. $|C(\theta)|<1$ if $\theta \neq 0$, and $C(0)=1$.

Wilf [1] has previously shown that there exists an integer $k_{0}$ such that Theorem 1 holds if $k \geqq k_{0}$, and has conjectured that $k_{0}=0$. The present proof, which is different from Wilf's, establishes the theorem for all $k \geqq 0$.

If a weighting function $w$ has a transform (2) which satisfies the inequality of Theorem 1, it is said to be stable, for reasons previously discussed by Schoenberg and DeForest, as described in [1] and its references. In addition, if one thinks of $w(x),-1 \leqq x \leqq 1$, as the weighting function of a filter with input

$$
f(x)=S(x)+N(x),
$$

where $S$ is a polynomial of degree not greater than $2 k+1$, and $N$ is a zero mean stationary random process with spectral density $\psi(\theta)$, then the output of the filter is

$$
\int_{-1}^{1} w(y) f(x-y) d y=S(x)+M(x),
$$

Presented to the Society, March 3, 1966; received by the editors April 4, 1966. 
where $M$ is a zero mean process with spectral density $C^{2}(\theta) \psi(\theta)$. Thus, the theorem guarantees that the filter (3) decreases the spectral density of the noise at every frequency.

\section{Proof of Theorem 1.}

LEMMA 1. There is a rational function of the form

$$
E(\theta)=1-\theta^{2 k+2}\left(\sum_{r=0}^{2 k+1} q_{r} \theta^{4 k-2 r+2}\right)^{-1}\left(\sum_{r=0}^{k} p_{r} \theta^{2 k-2 r}\right)
$$

which interpolates $C^{2}(\theta)$ at the critical points of $C(\theta)$. That is, if $C^{\prime}(\phi)=0$, then $E(\phi)=C^{2}(\phi)$.

Proof. Repeated integration by parts of (2) yields

$$
C(\theta)=A(\theta) \cos \theta+B(\theta) \sin \theta,
$$

with

$$
A(\theta)=\sum_{r=1}^{k}(-1)^{r-1} \gamma_{2 r-1} / \theta^{2 r}, \quad B(\theta)=\sum_{r=0}^{k}(-1)^{r} \gamma_{2 r} / \theta^{2 r+1},
$$

where $\gamma_{r}=2 w^{(r)}(1)$. Differentiating (5) yields

$$
C^{\prime}(\theta)=\left(A^{\prime}(\theta)+B(\theta)\right) \cos \theta+\left(B^{\prime}(\theta)-A(\theta)\right) \sin \theta .
$$

If we solve (5) and (6) for $\sin \theta$ and $\cos \theta$, square, and add the results, we are led to

$$
D^{2}=Q C^{2}+\left(A^{2}+B^{2}\right)\left(C^{\prime}\right)^{2}-2\left(A A^{\prime}+B B^{\prime}\right) C C^{\prime},
$$

where

$$
D=A\left(B^{\prime}-A\right)-B\left(A^{\prime}+B\right),
$$

and

$$
Q=\left(B^{\prime}-A\right)^{2}+\left(A^{\prime}+B\right)^{2} .
$$

Define

$$
E=D^{2} / Q \text {. }
$$

From (7), $E(\phi)=C^{2}(\phi)$ if $C^{\prime}(\phi)=0$. To see that $E$ has the form (4), consider the result of expanding both sides of (7) in powers of $\theta$. It is known, [2], that

$$
C(\theta)=1+\theta^{2 k+2} L(\theta),
$$

where $L$ is regular at $\theta=0$. (This is easily established by expanding 
(2) in powers of $\theta$ and integrating term by term, making use of the fact that if $f$ in (1) is a polynomial of degree not greater than $2 k+1$, then $p(0)=f(0)$.) Consequently $C^{\prime}(\theta)=\theta^{2 k+1} L_{1}(\theta)$, where $L_{1}$ is regular at $\theta=0$. Let

$$
R=Q-D^{2}
$$

and write (7) as

$$
\begin{aligned}
-R= & Q L \theta^{2 k+2}\left(2+\theta^{2 k+2} L\right)+\left(A^{2}+B^{2}\right)\left(C^{\prime}\right)^{2} \\
& -2\left(A A^{\prime}+B B^{\prime}\right) C C^{\prime} .
\end{aligned}
$$

$R$ contains only negative powers of $\theta$, and the largest power of $1 / \theta$ appearing on the right of (12) is $2 k+2$. Hence, $R$ can be written

$$
R(\theta)=\sum_{r=0}^{k} p_{r} \theta^{-2 r-2}
$$

Since $Q$ is of the form

$$
Q(\theta)=\sum_{r=0}^{2 k+1} q_{r} \theta^{-2 r-2}
$$

and $E=1-R / Q$, (4) now follows.

Also, we observe that $D$ is actually of the form

$$
D(\theta)=\sum_{r=0}^{k} d_{r} \theta^{-2 r-2}
$$

This is used below, but it is not obvious from (8), where it appears that $D$ could contain powers of $1 / \theta$ as high as $4 k+2$. However, from (11) and the fact that $Q$ is of degree only $4 k+4$ in $1 / \theta$, the presence of higher degree terms in $D$ would contradict (14).

Lemma 2. $0 \leqq E(\theta)<1$ if $\theta \neq 0$.

Proof. $E \geqq 0$ from (10), since $Q \geqq 0$. To establish the inequalities of the lemma, it is sufficient to show that $R>0$, since $E=1-R / Q$. We will show that the coefficients $p_{0}, \cdots, p_{k}$ in (13) are all positive. In this proof, some routine (although not always trivial) manipulations are omitted. Some of the relations are most easily established as special cases of identities which appear in the Appendix.

Define

$$
\lambda_{r}=\gamma_{r}+r \gamma_{r-1}, \quad \gamma_{-1}=0
$$

Then 


$$
B^{\prime}(\theta)-A(\theta)=-\sum_{r=0}^{k}(-1)^{r} \lambda_{2 r+1} \theta^{-2 r-2}
$$

and

$$
A^{\prime}(\theta)+B(\theta)=\sum_{r=0}^{k}(-1)^{r} \lambda_{2 r} \theta^{-2 r-1} .
$$

From (8) and (15),

$$
d_{r}=(-1)^{r+1} \sum_{s=0}^{2 r}(-1)^{s} \gamma_{s} \lambda_{2 r-s} \quad 0 \leqq r \leqq k .
$$

From (9) and (14),

$$
q_{r}=(-1)^{r} \sum_{s=0}^{2 r}(-1)^{s} \lambda_{s} \lambda_{2 r-s} \quad 0 \leqq r \leqq 2 k+1,
$$

and from (11) and (13),

$$
p_{0}=q_{0}
$$

and

$$
p_{r}=q_{r}-\sum_{s=0}^{r-1} d_{s} d_{r-s-1}, \quad 1 \leqq r \leqq k .
$$

Let $w(x)=w_{0}+w_{1} x^{2}+\cdots+w_{k} x^{2 k}$. Since

$$
\int_{-1}^{1} w(x) x^{2 i} d x=\delta_{0 i}, \quad 0 \leqq i \leqq k,
$$

it follows that

$$
\sum_{j=0}^{k}(2 i+2 j+1)^{-1} w_{j}=\delta_{0 i}, \quad 0 \leqq i \leqq k .
$$

The matrix of this system is essentially a Hilbert matrix. Its inverse has general element $a_{i j}$ given by

$$
a_{i j}=\frac{(-1)^{i+j}}{(k !)^{2}}\left(\begin{array}{l}
k \\
i
\end{array}\right)\left(\begin{array}{l}
k \\
j
\end{array}\right) \frac{\left(k+i+\frac{1}{2}\right)^{(k+1)}\left(k+j+\frac{1}{2}\right)^{(k+1)}}{\left(i+j+\frac{1}{2}\right)}
$$

where $(x)^{(r)}$ is the factorial polynomial. The polynomial $w$ is easily obtained, and its derivatives at $x=1$ evaluated to yield

$$
\gamma_{r}=\alpha \sum_{s=0}^{k}(-1)^{s}\left(\begin{array}{l}
k \\
s
\end{array}\right)\left(\begin{array}{c}
k+s+\frac{1}{2} \\
k
\end{array}\right)(2 s)^{(r)}
$$




$$
\lambda_{r}=\alpha \sum_{s=0}^{k}(-1)^{s}\left(\begin{array}{l}
k \\
s
\end{array}\right)\left(\begin{array}{c}
k+s+\frac{1}{2} \\
k
\end{array}\right)(2 s+1)^{(r)},
$$

where

$$
\alpha=2\left(k+\frac{1}{2}\right)^{(k+1)} / k !=2^{-2 k}(2 k+1) ! /(k !)^{2} .
$$

The quantity $\lambda_{r}$ can be written in closed form as

$$
\lambda_{r}=(-1)^{k} \frac{\alpha}{2^{r}} \frac{(2 k+r+1)^{(2 r)}}{r !},
$$

as is shown in the Appendix. To find $q_{r}$, substitute this in (17).

$$
q_{r}=(-1)^{r}\left(\alpha^{2} / 2^{2 r}(2 r) !\right) H_{r}(2 k+1),
$$

where $H_{r}(x)$ is defined by (A-1). (Equations designated by A- are in the Appendix.) Substituting $x=2 k+1$ in (A-2) yields

$$
q_{r}=2^{-2 r} \alpha^{2}\left(\begin{array}{c}
2 r \\
r
\end{array}\right)(2 k+r+1)^{(2 r)} .
$$

In particular, since $p_{0}=q_{0}, p_{0}$ is clearly positive. Hence, we need only show that $p_{1}, \cdots, p_{k}$ are positive. To express $d_{r}$, (16), in closed form, we use (19), (20), and the identity

$$
(2 s)^{(r)}=\sum_{j=0}^{r}(-1)^{r+j}(r)^{(r-j)}(2 s+1)^{(j)}
$$

to find that

$$
\gamma_{r}=\sum_{j=0}^{r}(-1)^{r+j}(r)^{(r-j)} \lambda_{j}
$$

Substituting this in (16) yields

$$
d_{r}=(-1)^{r+1} \alpha^{2} F(2 k+1),
$$

where $F(x)$ is defined by (A-3). This can be written in closed form (see Appendix) as

$$
d_{r}=(-1)^{r+1} 2^{2 r} \alpha^{2}(k)^{(r)}\left(-k-\frac{3}{2}\right)^{(r)} .
$$

Substituting this in (18) yields

$$
p_{r}=q_{r}-2^{2 r-2} \alpha^{4} \eta_{r-1}, \quad 1 \leqq r \leqq k,
$$

with 


$$
\begin{aligned}
\eta_{r} & =(-1)^{r} \sum_{s=0}^{r}(k)^{(s)}\left(-k-\frac{3}{2}\right)^{(s)}\left(-k-\frac{3}{2}\right)^{(r-s)}(k)^{(r-s)} \\
& =(-1)^{r} k !(k)^{(k-r)} \psi_{r}\left(-k-\frac{3}{2}\right),
\end{aligned}
$$

where

$$
\psi_{r}(x)=\sum_{s=0}^{r}\left(\begin{array}{l}
r \\
s
\end{array}\right) \frac{(x)^{(s)}(x)^{(r-s)}}{(k-s)^{(k-r)}(k-r+s)^{(k-r)}} .
$$

The expansion

$$
\begin{aligned}
\frac{1}{(k-r+s)^{(k-r)}(k-s)^{(k-r)}}= & \sum_{j=1}^{k-r} \frac{\left(\begin{array}{c}
2 k-2 r-j-1 \\
k-r-1
\end{array}\right)}{(2 k-r)^{(2 k-2 r-j)}} \\
& \cdot\left[\frac{1}{(s+j)^{(j)}}+\frac{1}{(r-s+j)^{(j)}}\right]
\end{aligned}
$$

derived in the Appendix, is useful here. Substituting it in (27), we find that

$$
\psi_{r}(x)=2 \sum_{j=1}^{k-r} \frac{\left(\begin{array}{c}
2 k-2 r-j-1 \\
k-r-1
\end{array}\right)}{(2 k-r)^{(2 k-2 r-j)}} \sum_{s=0}^{r}\left(\begin{array}{l}
r \\
s
\end{array}\right) \frac{(x)^{(s)}(x)^{(r-s)}}{(s+j)^{(j)}} .
$$

Now rewrite

(30)

$$
\begin{aligned}
\sum_{s=0}^{r}\left(\begin{array}{l}
r \\
s
\end{array}\right) & \frac{(x)^{(s)}(x)^{(r-s)}}{(s+j)^{(j)}} \\
= & \frac{1}{(x+j)^{(j)}(r+j)^{(j)}} \sum_{s=0}^{r}\left(\begin{array}{c}
r+j \\
s+j
\end{array}\right)(x+j)^{(s+j)}(x)^{(r-s)} \\
= & \frac{(2 x+j)^{(r+j)}}{(x+j)^{(j)}(r+j)^{(j)}} \\
& -\frac{1}{(x+j)^{(j)}(r+j)^{(j)}} \sum_{s=j+1}^{r}\left(\begin{array}{c}
r+j \\
s
\end{array}\right)(x+j)^{(s)}(x)^{(r+j-s)} .
\end{aligned}
$$

Substituting this in (29), we find that

$$
\psi_{r}(x)=\Gamma_{r}(x)+\xi_{r}(x),
$$

where 


$$
\begin{aligned}
\Gamma_{r}(x)= & 2 \sum_{j=0}^{k-r-1} \frac{\left(\begin{array}{c}
2 k-2 r-j-2 \\
k-r-1
\end{array}\right)}{(2 k-r)^{(2 k-2 r-j-1)}} \\
& \frac{(2 x+j+1)^{(r+j+1)}}{(r+j+1)^{(j+1)}(x+j+1)^{(j+1)}} .
\end{aligned}
$$

We have no interest in $\xi_{r}$, except to note that

$$
(-1)^{r+1} \xi_{r}\left(-k-\frac{3}{2}\right)>0,
$$

which can be seen by inspection of the last member on the right of (30). After some manipulation, $\Gamma_{r}$ can be written

$$
\begin{aligned}
& \Gamma_{r}(x)=\frac{2(2 x+1)^{(r+1)}}{(x+k-r)^{(k-r)}(2 k-r)^{(2 k-2 r)}} \\
& \quad \cdot \sum_{j=0}^{k-r-1}\left(\begin{array}{c}
2 k-2 r-j-2 \\
k-r-1
\end{array}\right)(2 x+j+1)^{(j)}(x+k-r)^{(k-r-j-1)}
\end{aligned}
$$

The sum can be written in closed form by referring to (A-6) with $m=k-r-1$, and reversing the order of summation. The result is

$$
\Gamma_{r}(x)=\frac{2^{k-r}(2 x+1)^{(r+1)} \prod_{j=1}^{k-r-1}(2 x+2 j+1)}{(x+k-r)^{(k-r)}(2 k-r)^{(2 k-2 r)}} .
$$

From (26), (31), and (32),

$$
\eta_{r}<(-1)^{r} k !(k)^{(k-r)} \Gamma_{r}\left(-k-\frac{3}{2}\right) .
$$

Hence, from (25), with $r$ replaced by $r+1$,

$$
p_{r+1}>q_{r+1}-(-1)^{r} 2^{2 r} \alpha^{4} k !(k)^{(k-r)} \Gamma_{r}\left(-k-\frac{3}{2}\right),
$$

if $0 \leqq r=k-1$. By routine manipulations, using this and (22), the right side can be shown to vanish. This completes the proof of Lemma 2, and Theorem 1 is now evident.

3. Appendix. a. Derivation of (21). We indicate the proof for even $r$. Write

$$
(2 s+1)^{(2 m)}=2^{2 m}\left(s+\frac{1}{2}\right)^{(m)}(s)^{(m)}
$$

and substitute in (20) to obtain

$$
\lambda_{2 m}=2^{2 m} \alpha(k+m)^{(2 m)} \sum_{s=m}^{k}(-1) \cdot\left(\begin{array}{c}
k-m \\
s-m
\end{array}\right)\left(\begin{array}{c}
k+q+\frac{1}{2} \\
k+m
\end{array}\right) .
$$


The sum can be recognized as the $(k-m)$ th difference of $C_{x, m+k}$ at $x=k+m+\frac{1}{2}$, except for the factor $(-1)^{k}$. Hence

$$
\lambda_{2 m}=(-1)^{k} 2^{2 m} \alpha(k+m)^{(2 m)}\left(\begin{array}{c}
k+m+\frac{1}{2} \\
2 m
\end{array}\right),
$$

which can be reduced to (21), with $r=2 m$, by observing that

$$
(k+m)^{(2 m)}\left(k+m+\frac{1}{2}\right)^{(2 m)}=2^{-4 m}(2 k+m+1)^{(4 m)} .
$$

b. Derivation of (22). Define

$$
H_{r}(x)=\sum_{s=0}^{2 r}(-1)^{s}\left(\begin{array}{c}
2 r \\
s
\end{array}\right)(x+s)^{(2 s)}(x+2 r-s)^{(4 r-2 s)} .
$$

If we expand $(x+s)^{(2 s)}(x+2 r-s)^{(4 r-2 s)}$ in powers of $x$, it is easy to verify that the coefficient of $x^{4 r-m}$ is a polynomial in $s$ of degree not greater than $m$, so that its $2 r$ th difference vanishes if $0 \leqq m \leqq 2 r-1$. Thus, the degree of $H_{r}$ does not exceed $2 r$. Each term on the right of $(\mathrm{A}-1)$ has $(x+r)^{(2 r)}$ as a factor. Hence

$$
H_{r}(x)=(-1)^{r}\left(\begin{array}{c}
2 r \\
r
\end{array}\right)(2 r) !(x+r)^{(2 r)},
$$

where the constant factor is obtained by setting $x=r$ in (A-1).

c. Derivation of (24). Consider

$$
\begin{aligned}
F(x)= & \sum_{m=0}^{2 r} \frac{2^{-m}}{m !} \sum_{j=0}^{m}(-1)^{j}\left(\begin{array}{c}
m \\
j
\end{array}\right)(2 r-m+j)^{(2 r-m)}(x+j)^{(2 j)} \\
& \cdot(x+m-j)^{(2 m-2 j)} .
\end{aligned}
$$

Since $d_{r}=0$ if $r>k$, (see remark following Lemma 1), we conclude from (23) that $F(2 k+1)=0$ if $0 \leqq k<r$, from which we infer that $F(x)=0$ if $x=1,3, \cdots, 2 r-1$. Furthermore, it can be shown that $F(-x-1)=F(x)$, by substituting $-x-1$ for $x$ in (A-3), replacing $j$ by $m-j$ in the first summation, and using the identity $(y)^{(n)}$ $=(-1)^{n}(-y+n-1)^{(n)}$. Hence, $F$ also has the roots $x=-2,-4, \cdots$, $-2 r$. Furthermore, $F$ is actually a polynomial of degree $2 r$, (see the remark above concerning the degree of $\left.H_{r}\right)$, and $F(0)=(2 r)$ !. Hence

$$
F(x)=(-1)^{r}(x-1) \cdots(x-2 r+1)(x+2) \cdots(x+2 r) .
$$

To obtain (24), set $x=2 k+1$.

d. Derivation of (28). We start by establishing the identity 


$$
\begin{aligned}
\sum_{i=0}^{m}\left(\begin{array}{c}
m+i \\
i
\end{array}\right)(y-i)^{(m-i)}\left[(x+m+1)^{(i)}(y-x)^{(m+1)}\right. & \\
& \left.+(x+m+1)^{(m+1)}(y-x)^{(i)}\right]=(y+m+1)^{(2 m+1)} .
\end{aligned}
$$

Consider the left side to be $G$, a polynomial of degree $2 m+1$ in $x$. It is straightforward to show that

$$
G(y-j)=(y+m+1)^{(2 m+1)}, \quad 0 \leqq j \leqq m .
$$

Since both sides of (A-4) are unchanged if $x$ is replaced by $y-x-m-1$, we can infer that (A-4) holds for $2 m+2$ distinct values of $x$, and is an identity. Equation (28) can be obtained by dividing (A-4) by

$$
(y+m+1)^{(2 m+1)}(x+m+1)^{(m+1)}(y-x)^{(m+1)},
$$

setting $x=s, y=k, m=k-r-1$, and then replacing $i$ by $j=k-r-i$.

e. Derivation of (34). Set $y=2 x+m+1$ in (A-4) to obtain

$$
\begin{gathered}
\sum_{i=0}^{m}\left(\begin{array}{c}
m+i \\
m
\end{array}\right)(2 x+m-i+1)^{(m-i)}(x+m+1)^{(i)} \\
=2^{m} \prod_{j=1}^{m}(2 x+2 j+1) .
\end{gathered}
$$

To obtain (34), we must evaluate the sum in (33), which can be done by setting $m=k-r-1$ in (A-6), and reversing the order of summation.

4. Added in proof. Lorch and Szego [3] have discovered and corrected in error in [1]. They have also proved Theorem 1 for all $k \geq 0$, by a method different from that presented here [4].

Greville [5] has considered the analogous problem in the theory of smoothing discrete data.

\section{REFERENCES}

1. H. S. Wilf, The stability of smoothing by least squares, Proc. Amer. Math. Soc. 15 (1964), 933-937.

2. I. J. Schoenberg, On smoothing operations and their generating functions, Bull. Amer. Math. Soc. 59 (1953), 199-230.

3. L. Lorch and P. Szego, $A$ Bessel function inequality connected with the stability of least square smoothing, Proc. Amer. Math. Soc. 17 (1966), 330-332.

4. Private correspondence.

5. T. N. E. Greville, On the stability of linear smoothing formulas, J. Soc. Indust. Appl. Math. Ser. B. Numer. Anal. 3 (1966), 157-170.

Drexel Institute of Technology 